PROCEEDINGS OF THE

AMERICAN MATHEMATICAL SOCIETY

Volume 138, Number 12, December 2010, Pages 4443-4446

S 0002-9939(2010)10446-X

Article electronically published on June 22, 2010

\title{
ONE-PARAMETER FAMILIES OF SMOOTH INTERVAL MAPS: DENSITY OF HYPERBOLICITY AND ROBUST CHAOS
}

\author{
SEBASTIAN VAN STRIEN \\ (Communicated by Yingfei Yi)
}

\begin{abstract}
In this paper we will discuss the notion of robust chaos and show that (i) there are natural one-parameter families of interval maps with robust chaos and (ii) hyperbolicity is dense within generic one-parameter families (and so these families are not robustly chaotic).
\end{abstract}

\section{Statement of Results}

In [5] the notion of robust chaos was introduced. A family of maps $\left\{f_{t}\right\}_{t \in[0,1]}$ is said to have robust chaos (or to be robustly chaotic) if there exists no parameter $t \in[0,1]$ for which the map $f_{t}$ has a periodic attractor. Examples of families with robust chaos where given in that paper, but in these families the maps are nonsmooth. The authors suggest that 'it is known that robust chaos cannot occur in smooth systems', but this turns out to be not true. Indeed, in [3, 2, [11 [8] and [1], examples where given of families of smooth one-dimensional maps with robust chaos. Since there is a huge body of literature on bifurcations of one-parameter families of dynamical systems (starting with, for example, 12]), we shall clarify the situation in this paper.

1.1. Theorem (Robust unimodal families are 'constant'). Let $\left\{f_{t}\right\}$ be a family of unimodal maps with robust chaos. Assume this family satisfies the following regularity conditions: (i) $\quad(x, t) \mapsto f_{t}(x)$ is $C^{0}$ and (ii) for each $t$, the map $x \mapsto$ $f_{t}(x)$ is $C^{1}$, has precisely one critical point and has no wandering intervals. Then all maps within this family are topologically conjugate.

So the family of robustly chaotic unimodal maps given in the papers cited above are all topologically conjugate to each other. That the family is robustly chaotic is therefore not surprising!

Note that the assumption that $f_{t}$ has no wandering intervals is rather mild: it suffices that for each $t, x \mapsto f_{t}(x)$ is $C^{2}$ and that $f_{t}$ is non-flat near the critical point $c_{t}$. This means that there exists a $C^{2}$ local diffeomorphism $\phi_{t}$ with $\phi_{t}\left(c_{t}\right)=0$ such that $f_{t}(x)= \pm\left|\phi_{t}(x)\right|^{\alpha}+f_{t}(c)$ for some $\alpha \geq 2$. This holds for example when $x \mapsto f_{t}(x)$ is real analytic. For a proof of this and for a proof of even weaker conditions for this to hold, see [6], 7] and [15].

The next theorem shows that there are robustly chaotic multimodal families which are not 'topologically constant'.

Received by the editors December 3, 2009 and, in revised form, February 15, 2010.

2010 Mathematics Subject Classification. Primary 37E05, 37Gxx, 37Dxx.

(c) 2010 American Mathematical Society Reverts to public domain 28 years from publication 
1.2. Theorem (A family of cubic maps with robust chaos). There exists a oneparameter family $\left\{f_{t}\right\}$ of interval maps (consisting of cubic polynomials) which is robustly chaotic and such that not all maps within this family are topologically conjugate.

In fact, for any $d \geq 3$ there exists a one-parameter family of interval maps consisting of polynomials of degree $d \geq 3$ which is robustly chaotic (and not topologically constant).

On the other hand, the above example is special: generic one-parameter families are not robustly chaotic. In fact, hyperbolicity is dense within such families, i.e., every critical point is in the basin of a periodic attractor.

1.3. Theorem (Only exceptional families are robustly chaotic). Let $0 \leq r \leq \infty$ and $2 \leq k \leq \infty$. For a generic $C^{r}$ one-parameter family $\left\{f_{t}\right\}_{t \in[0,1]}$ of $C^{\bar{k}}$ interval maps with $d$ critical points the following properties hold:

(a) the number of critical points of each of the maps $f_{t}$ is bounded;

(b) the set of parameters $t$ for which

(i) all critical points of $f_{t}$ are in basins of periodic attractors (such a map $f_{t}$ is called hyperbolic),

(ii) each critical point of $f_{t}$ is non-degenerate (i.e. quadratic),

(iii) critical points of $f_{t}$ are not eventually mapped onto other critical points (such a map $f_{t}$ is said to have 'no critical relations')

is open and dense.

In other words, we assume that $\partial^{i+j} /\left(\partial t^{i} \partial x^{j}\right) f_{t}(x)$ exists and is continuous in $(t, x)$ for all integers $i \leq r$ and $j \leq k$ and we consider the corresponding topology of uniform convergence. In fact, if $r \geq 1$ and $k \geq 3$, then by elementary catastrophy theory (see for example [4]), for generic one-parameter families of functions $\left\{f_{t}\right\}_{t \in[0,1]}$ there are only a finite number of parameters $0<t_{1}<t_{2}<\cdots<t_{n}<1$ for which $f_{t}$ has a degenerate critical point. At these parameters $t$, one of the critical points of $f_{t}$, say $c$, is cubic and undergoes a 'fold catastrophe'.

We should also point out the following two facts; see [7]. If $f_{t}$ is hyperbolic, then Lebesgue almost every $x$ is in the basin of a hyperbolic periodic attractor of $f_{t}$. If, in addition, $f_{t}$ has only non-degenerate critical points and no critical relations, then it is structurally stable (at this parameter). So Theorem 1.3 implies that for generic one-parameter families as above, the set of structurally stable parameters is open and dense.

Theorem 1.3 also holds within the space of families of polynomial interval maps: families satisfying (a) and (b) are generic (i.e. of 2nd Baire category); see the very last paragraph of this paper. Theorem 1.3 is based on [9] and [10], which show density of hyperbolicity within the space of real polynomials. Using [13] one can prove the analogous result within the space of certain families of entire transcendental maps. For a survey on related results, see [14].

\section{The PROOFS}

Let us start with the proof of Theorem 1.1. Take a robustly chaotic family $\left\{f_{t}\right\}$ of unimodal maps $f_{t}:[0,1] \rightarrow[0,1]$. Since $f_{t}$ is assumed to have only one critical point, the turning point $c_{t}$ depends continuously on $t$. The itinerary of the turning point $c_{t}$ of $f_{t}$ can change only as $t$ varies if $f_{t}^{n}\left(c_{t}\right)=c_{t}$ for some $n$ and for some $t=t_{0}$. But since $x \mapsto f_{t}(x)$ is $C^{1}$ this implies that $c_{t}$ is a periodic attractor 
(with multiplier 0 ) of $f_{t}$ when $t=t_{0}$. Since $\left\{f_{t}\right\}$ is robustly chaotic, this does not happen. So $f_{t}$ has the same kneading invariant for each $t \in[0,1]$. Since $f_{t}$ has no periodic attractors at all, it follows from the non-existence of wandering intervals (see Chapter IV of [7]) that $f_{t^{\prime}}$ and $f_{t}$ are topologically conjugate for all $t, t^{\prime} \in[0,1]$.

Let us now prove Theorem 1.2 and show that there exists a family of cubic maps with robust chaos and which does not have constant kneading invariant. Consider polynomials $f:[0,1] \rightarrow[0,1]$ of degree three, so that $f(0)=0, f(1)=1$ (which implies that $\left.f(x)=a x+b x^{2}+(1-a-b) x^{3}\right)$ and with two critical points $0<$ $c_{1}<c_{2}<1$ so that $0<c_{1}<f\left(c_{2}\right)<f^{3}\left(c_{2}\right)=f^{4}\left(c_{2}\right)<c_{2}<f^{2}\left(c_{2}\right)<f\left(c_{1}\right)<1$. The set of such polynomials corresponds to a real analytic curve in the $(a, b)$-plane (defined by the condition that $f^{4}\left(c_{2}\right)=f^{3}\left(c_{2}\right)$ ). Hence it contains a one-parameter family of maps $\left\{f_{t}\right\}_{t \in[0,1]}$. Since $f_{t}$ is a polynomial with only real critical points, it has negative Schwarzian (see [7, Exercise IV.1.7]). Hence by Singer's result, each of its periodic attractors has a critical point in its immediate basin. Since $\left[f\left(c_{2}\right), 1\right]$ is mapped into itself and $f\left(c_{1}\right) \in\left[f\left(c_{2}\right), 1\right]$, any periodic attractor of $f_{t}$ would have to lie in $\left[f\left(c_{2}\right), 1\right]$. Since $c_{2}$ is the only critical point in $\left[f\left(c_{1}\right), 1\right]$, it follows that if $f_{t}$ has a periodic attractor, then $c_{2}$ would have to be in its basin. But since $f^{4}\left(c_{2}\right)=f^{3}\left(c_{2}\right)$ is a repelling fixed point, this does not happen. It follows that these maps define a one-parameter family $\left\{f_{t}\right\}$ of smooth bimodal maps which are robustly chaotic. Since $f_{t}\left(c_{1}\right)$ can vary with $t$ (to be anywhere within the interval $\left.\left[\left(f_{t}\right)^{2}\left(c_{2}\right), 1\right]\right)$, the kneading invariant of $f_{t}$ is not constant. Note that this example is based on the map having a trapping region.

Let us finally prove Theorem 1.3. When $2 \leq k<\infty$, let $H$ be the Banach space of $C^{k}$ maps $f:[0,1] \rightarrow[0,1]$ (endowed with the $C^{k}$ supremum norm). When $k=\infty$, let $H$ be the corresponding Fréchet space of $C^{\infty}$ maps $f:[0,1] \rightarrow[0,1]$. By [10] (which is based on 9]) there exists an open and dense subset $X^{\prime} \subset H$ so that each $f \in X^{\prime}$ is hyperbolic. Maps for which the critical points are degenerate or which have critical relations correspond to a countable union of analytic codimension-one varieties. So we can find an open and dense subset $X \subset X^{\prime}$ such that each $f \in X$ satisfies conditions (b)(i), (b)(ii) and (b)(iii) of Theorem 1.3.

Let $B$ be an open neighbourhood of the zero function in the Banach (or Fréchet space) of $C^{k}$ functions $\alpha:[0,1] \rightarrow \mathbb{R}$. We can identify a one-parameter family $\left\{f_{t}\right\}$ with a curve $c:[0,1] \rightarrow H$. Hence Theorem 1.3 follows from

2.1. Lemma. Let $H$ be a Banach space or a Fréchet space, let $B \subset H$ be an open subset of $H$, and let $c:[0,1] \rightarrow H$ be a curve. Let $X$ be an open and dense subset of $H$. Then there exists a set $A \subset B$ which is dense in $B$ (in fact of 2 nd Baire category) so that for each $\alpha \in A, F_{\alpha}:=\{t \in[0,1] ; c(t)+\alpha \in X\}$ is open and dense.

Proof. Since the curve $c$ is continuous and $X$ is open, $F_{\alpha}$ is open for each $\alpha \in B$. Let us construct a set $A$ with the property that $F_{\alpha}$ is dense for each $\alpha \in A$. To do this, take $\delta>0$ and define the set $A_{\delta}$ of $\alpha \in B$ so that for each $t \in[0,1]$ there exists $t^{\prime}$ with $\left|t-t^{\prime}\right|<\delta$ and so that $t^{\prime} \in F_{\alpha}$.

Let us show that $A_{\delta}$ is dense. Assume by contradiction it is not dense. Then there exists an open set $U$ of $\alpha \in B$ for which there exists $t_{\alpha} \in[0,1]$ so that for each $t \in[0,1]$ with $\left|t-t_{\alpha}\right|<\delta$ one has $t \notin F_{\alpha}$. So if we take $m>1 / \delta$, then for each $\alpha \in U$ there exists $k \in\{0,1, \ldots, m\}$ so that $k / m \notin F_{\alpha}$, i.e. $c(k / m)+\alpha \notin X$. Let $U_{k}$ be the set of $\alpha \in U$ so that $c(k / m)+\alpha \notin X$. Note that $U_{0} \cup \cdots \cup U_{m}=U$. It follows that the closure of at least one of the sets $U_{k_{0}}$ contains an open set (otherwise $U-\bar{U}_{i}$ is dense in $U$ for each $i$, and so by the Baire property $\bigcap_{i=0, \ldots, m}\left(U-\bar{U}_{i}\right)=U-\bigcup_{i=0, \ldots, m} \bar{U}_{i}$ is 
dense in $U$, a contradiction). Note that for each $\alpha \in U_{k_{0}}$ one has $c\left(k_{0} / m\right)+\alpha \notin X$. But since $X$ is open, then for each $\alpha \in \overline{U_{k_{0}}}$ one has $c\left(k_{0} / m\right)+\alpha \notin X$. But this and the fact that $\overline{U_{k_{0}}}$ contains an open set contradict the assumption that $X$ is open and dense. Thus we have shown that $A_{\delta}$ is dense for each $\delta>0$.

Since $A_{\delta}$ is also open, it follows by the Baire property that $A:=\bigcap_{\delta>0} A_{\delta}$ is dense. By construction, for each $\alpha \in A$, we have that $F_{\alpha}$ is dense.

The analogue of Theorem 1.3 also holds in the case of one-parameter families of polynomial maps of degree $d$. Indeed, in that case let $F$ be the space of all real polynomial interval maps of degree $d$. Again by [9] and [10] there exists an open and dense subset $X^{\prime}$ of $F$ consisting of real hyperbolic polynomials. As before, conditions (b)(ii) and (b)(iii) correspond to analytic codimension-one varieties. Thus the proof above applies also to the polynomial case.

\section{REFERENCES}

1. J.M. Aguirregabiria, Robust chaos with variable Lyapunov exponent in smooth onedimensional maps, Chaos, Solitons and Fractals 42 (2009), no. 4, 2531-2539.

2. M. Andrecut and M.K. Ali, Example of robust chaos in a smooth map, Europhysics Letters 54 (2001), no. 3, 300-305.

3. Robust chaos in smooth unimodal maps, Physical Review E 64 (2001), no. 2, 25203. MR.1849767 (2002e:37048)

4. V. I. Arnol'd, Catastrophe theory, third ed., Springer-Verlag, Berlin, 1992, translated from the Russian by G. S. Wassermann, based on a translation by R. K. Thomas. MR.1178935 (93h:58018)

5. S. Banerjee, J.A. Yorke, and C. Grebogi, Robust chaos, Physical Review Letters 80 (1998), no. 14, 3049-3052.

6. Welington de Melo and Sebastian van Strien, A structure theorem in one-dimensional dynamics, Ann. of Math. (2) 129 (1989), no. 3, 519-546. MR90m:58106

7. __ One-dimensional dynamics, Ergebnisse der Mathematik und ihrer Grenzgebiete (3) [Results in Mathematics and Related Areas (3)], vol. 25, Springer-Verlag, Berlin, 1993. MR95a:58035

8. Z. Elhadj and J.C. Sprott, On the robustness of chaos in dynamical systems: Theories and applications, Frontiers of Physics in China 3 (2008), no. 2, 195-204.

9. O.S. Kozlovski, W. Shen, and S. van Strien, Rigidity for real polynomials, Ann. of Math. (2) 165 (2007), no. 3, 749-841. MR2335796 (2008m:37063)

10. , Density of hyperbolicity in dimension one, Ann. of Math. (2) 166 (2007), no. 1, 145-182. MR2342693 (2008j:37081)

11. E. Lett, Example of robust chaos in a smooth map, Europhysics Letters 54 (2001), no. 3, 300-305.

12. S. Newhouse, J. Palis, and F. Takens, Bifurcations and stability of families of diffeomorphisms, Inst. Hautes Études Sci. Publ. Math. 57 (1983), 5-71. MR699057(84g:58080)

13. Lasse Rempe and Sebastian van Strien, Density of hyperbolicity for real transcendental entire functions with real singular values, in preparation, 2010.

14. Sebastian van Strien, One-dimensional dynamics in the new millennium, Discr. and Cont. Dyn. Syst. A 27 (2010), no. 2, 557-588.

15. Sebastian van Strien and Edson Vargas, Real bounds, ergodicity and negative Schwarzian for multimodal maps, J. Amer. Math. Soc. 17 (2004), no. 4, 749-782 (electronic). MR2083467

Mathematics Institute, University of Warwick, Coventry CV4 7AL, United Kingdom E-mail address: strien@maths.warwick.ac.uk 